\title{
Vividness, spatial manipulation, and spontaneous elaboration: A critical evaluation of the use of factor analysis by Lorenz and Neisser (1985)
}

\author{
JOHN T. E. RICHARDSON \\ Brunel University, Uxbridge, Middlesex, England
}

\begin{abstract}
On the basis of factor analyses of questionnaire responses and psychometric test performance, Lorenz and Neisser (1985) argued for the existence of three distinct imagery factors: vividness and control, spatial manipulation, and spontaneous elaboration. Their use of factor analysis is criticized under three headings: the choice of the number of factors to be extracted, the choice of analytic model, and the choice of rotation model. On the basis of alternative analyses, it is concluded that the results of Lorenz and Neisser's investigation tend to support the established distinction between introspective judgments of experienced imagery and objective performance in tests of spatial thinking, but that there is no evidence for a third factor of spontaneous elaboration.
\end{abstract}

Mental imagery is both a subjective experience and a cognitive representation whose use may facilitate performance in a variety of objective tasks. Nevertheless, factoranalytic studies using psychometric instruments designed to study the vividness of experienced imagery and the capacity for spatial visualization have concluded that these represent distinct and possibly independent traits of individual subjects (Di Vesta, Ingersoll, \& Sunshine, 1971; Forisha, 1975; Richardson, 1972, 1977a; cf. Paivio, 1971, p. 496). A recent paper in the same tradition by Lorenz and Neisser (1985) confirmed this distinction and argued for the existence of yet another dimension, which they described as "spontaneous elaboration." The latter dimension was derived from earlier work by Slee (1980) and Garro (1983), and represented a "tendency to visualize details of the appearance of an object (as distinct from its identity or meaning) even when no specific task requires it" (Lorenz \& Neisser, 1985, p. 494). Lorenz and Neisser found that none of these three imagery factors was significantly correlated with the strength of childhood memories. They discussed a number of methodological considerations that might have obscured such relationships, but they did not address the more basic question of the adequacy of their analytic techniques. In this paper I shall critically evaluate those techniques in the light of conventional statistical wisdom and present further analyses that tend to qualify some of the conclusions reached by Lorenz and Neisser. These analyses were carried out upon the triangular matrix of correlation coefficients shown in Lorenz and Neisser's

I am grateful to Alice Healy and Ulric Neisser for their comments on a previous version of this paper, which was written while I was visiting the Open University as Honorary Senior Research Fellow. Requests for reprints should be sent to John T. E. Richardson, Department of Human Sciences, Brunel University, Uxbridge, Middlesex UB8 3PH, England.
(1985) Table 2, using the package SPSS/PC+ (Norusis, 1986).

\section{LORENZ AND NEISSER'S METHOD}

Lorenz and Neisser (1985) administered a battery of psychometric tests to a sample of students at Cornell University. Seven measures were obtained from tests concerned with mental imagery: the shortened version of Betts's (1909) Questionnaire Upon Mental Imagery developed by Sheehan (1967); the revised version of Gordon's (1949) test of imagery control that was published by Richardson (1969); the Vividness of Visual Imagery Questionnaire devised by Marks $(1972,1973)$; the VerbalizerVisualizer Questionnaire developed by Richardson (1977b); a Cube Task devised by Richardson (1977a) in which subjects are required to answer questions about an imagined cube that has been divided into 27 smaller cubes by two equidistant horizontal cuts, two equidistant vertical cuts, and two equidistant lateral cuts; the Differential Aptitude Test (DAT), Space Relations Form T (Bennett, Seashore, \& Wesman, 1947); and versions of Barratt's (1953) Visualization Form in which the subjects reported on the vividness, relevance, and controllability of images experienced while completing the DAT and the Cube Task. Six measures were obtained from a Recalled-Event Description task: four reflected the richness of autobiographical memories of specific events (the previous January 1, the day President Reagan was shot, the subject's first day at school, and the first manned landing on the moon), and two measured the subject's age at the time of the earliest personal memory and the richness of that memory (as indicated by the number of written words used to describe it). Finally, two measures were obtained from a separate task in which the subjects were asked to recall personal experiences before they were 6 years old: one was the mean number of memories listed, the other was the subject's mean age at the time of the recalled events.

The total sample of subjects consisted of 58 students, although 12 of these failed to provide complete sets of data, and only the remainder contributed to the main analysis of results. Of course, a final sample of $\mathbf{4 6}$ subjects is a relatively modest basis for a factor analysis, especially when a total of 15 variables is under consideration.

\section{BASIC ANALYSIS}

Lorenz and Neisser (1985) reported that a principal components analysis of all 15 variables was used to extract three 
factors, and that Varimax rotation yielded factors that could readily be identified in the following manner: Factor 1 , vividness and control; Factor 2, spatial manipulation; and Factor 3, childhood memory. Table 1 shows the results of an identical analysis carried out upon the matrix of correlation coefficients contained in Lorenz and Neisser's Table 2. Allowing for rounding errors inherent in the latter table, these results confirm the findings presented in their original paper. There are, nevertheless, three important issues to be addressed in evaluating these results: the number of factors to be extracted, the use of principal components analysis, and the use of Varimax rotation.

With regard to the first of these, Lorenz and Neisser reported that they decided to extract three factors on the basis of "inspection of the pattern of significant correlations" (p. 497). However, a matrix of simple correlation coefficients simply does not provide a perspicuous view of the relationships among a set of variables, and cannot be relied upon to guide analytic judgments concerning their internal structure. The whole point of factor analysis is to make that structure explicit. Although there is no generally agreed-upon criterion for deciding the number of factors to be extracted, two commonly used rules of thumb are the number of factors whose eigenvalues are greater than one in a principal components analysis (Kaiser, 1960), and the number of factors up to the point where the difference between successive eigenvalues in a principal components analysis reflects a relatively constant increment attributable to random error (the "scree test": Cattell, 1966). For the results reported by Lorenz and Neisser, the eigenvalues for 10 successive factors obtained from principal components analysis were $2.83,2.34,2.10,1.19,1.18$, $1.04, .87, .72, .63$, and .57 . A mechanical application of Kaiser's eigenvalue-one criterion suggested that six factors might be extracted, but Cattell's scree test indicated that Lorenz and Neisser's choice of three factors was in fact rather more suitable.

Nevertheless, with regard to the second point, there are reasons to question the appropriateness of principal com- ponents analysis for the sort of investigation conducted by Lorenz and Neisser. This sort of analysis proceeds by assigning the variance associated with the original variables to as many orthogonal dimensions or components. From a practical point of view, it is based upon the original correlation matrix among the variables in question, in which the diagonal elements have a value of 1.00 by definition. This is tantamount to assuming that the common variance to be explained is $100 \%$ of the total variance on each variable, or, in other words, that there is no variance unique to each variable and that every variable is perfectly reliable. Assumptions of this sort are highly questionable in the case of the measures that were considered by Lorenz and Neisser. Indeed, only one of the final communalities reported in their Table 3 is greater than .7, and several are less than .5 .

In contrast, common factor analysis is concerned only with the variance that is common to two or more of the variables in question, and explicitly excludes from consideration the variance that is unique to one single variable or merely the result of random error. From a practical point of view, it is based upon an amended correlation matrix in which the diagonal elements are replaced by estimates of the communality of the corresponding variables, and thus acknowledges the fact that the other elements in that matrix are necessarily reduced by test-retest unreliability. It is well known that the choice of communality estimates can make a substantial difference to the factorial solution that is eventually obtained (e.g., Rummel, 1970, pp. 313-315). However, most authorities recommend an iterative solution based upon the use of the squared multiple correlation (SMC) of each variable with all of the others as an initial estimate of communality. For the correlation matrix presented by Lorenz and Neisser, 10 of the 15 SMCs were less than .5 , reinforcing the point that one can expect only a fraction of the total variance to be explained by means of factor analysis. Table 1 shows the results of Varimax rotation of three factors extracted by common factor analysis from the matrix of correlation coefficients contained

Table 1

Results of Varimax Principal Components Analysis (PCA) and of Varimax and Oblimin Common Factor Analysis (CFA) of All Variables

\begin{tabular}{|c|c|c|c|c|c|c|c|c|c|}
\hline & \multicolumn{3}{|c|}{ Varimax PCA } & \multicolumn{3}{|c|}{ Varimax CFA } & \multicolumn{3}{|c|}{ Oblimin CFA } \\
\hline & 1 & 2 & 3 & 1 & 2 & 3 & 1 & 2 & 3 \\
\hline Betts (1909) & $.78 *$ & -.09 & -.12 & $.74 *$ & -.12 & -.13 & $.73 *$ & -.08 & -.11 \\
\hline Gordon (1949) & $.78 *$ & .17 & -.10 & $.74^{*}$ & .12 & -.10 & $.74^{*}$ & .17 & -.09 \\
\hline Marks $(1972,1973)$ & $.55^{*}$ & .18 & .23 & .46 & .11 & .17 & .47 & .12 & .18 \\
\hline Richardson (1977a) & -.01 & $.83^{*}$ & -.02 & .03 & $.78 *$ & -.02 & .05 & $.78 *$ & -.05 \\
\hline Barratt (1953) & .32 & $.80 *$ & .10 & .36 & $.78 *$ & .10 & .39 & $.79 *$ & .08 \\
\hline Bennett et al. (1947) & -.17 & $.71^{*}$ & -.29 & -.12 & $.63^{*}$ & -.26 & -.10 & $.63 *$ & -.28 \\
\hline Mean Age & -.17 & -.10 & $.79 *$ & -.16 & -.10 & $.76^{*}$ & -.16 & -.15 & $.76^{*}$ \\
\hline Earliest Age & .28 & .05 & $.74^{*}$ & .27 & .03 & $.68^{*}$ & .27 & .01 & $.69 *$ \\
\hline School & .32 & -.16 & $-.66^{*}$ & .25 & -.12 & $-.55 *$ & .24 & -.08 & $-.54 *$ \\
\hline Moon & -.18 & .12 & $-.60 *$ & -.14 & .11 & -.46 & -.14 & .12 & -.47 \\
\hline January 1 & .47 & -.44 & .03 & .33 & -.33 & .04 & .32 & -.31 & .06 \\
\hline Reagan & -.31 & -.02 & .07 & -.21 & -.03 & .05 & -.21 & -.04 & .04 \\
\hline Earliest Memory & .24 & -.01 & .15 & .16 & .01 & .10 & .17 & .01 & .11 \\
\hline Richardson (1977b) & .40 & .49 & .07 & .35 & .38 & .05 & .37 & .39 & .04 \\
\hline Memory Number & .27 & .26 & -.35 & .22 & .19 & -.26 & .22 & .22 & -.26 \\
\hline
\end{tabular}

*Loadings greater than .50 were used to interpret the factors. 
in Lorenz and Neisser's Table 2. The factor loadings are not dissimilar to those obtained in the previous analysis, suggesting that principal components analysis, although inappropriate, is not in this case misleading.

In their discussion, Lorenz and Neisser emphasized the point that the factors identified in their analysis are distinct from one another. To be sure, both of the analyses discussed thus far have implied that they reflect different traits that are orthogonal to one another. However, the latter is merely an artifact of imposing a Varimax (i.e., orthogonal) rotation upon the original factor matrix, and Lorenz and Neisser did not explore the validity of a solution obtained by means of oblique rotation. It is in fact entirely plausible that the different traits in question might be produced by overlapping sets of psychological processes. In this case, the resulting dimensions might be expected to be correlated with one another, and an oblique rotation of the extracted factor matrix would be far more appropriate (see Cattell, 1978, p. 128). Table 1 shows the structure factor matrix from an oblimin rotation (Carroll, 1957; Rummel, 1970, pp. 414-416) carried out upon the three factors extracted by means of common factor analysis from the matrix of correlation coefficients contained in Lorenz and Neisser's Table 2. The intercorrelations among the three factors were as follows: Factors 1 and $2,+.09$; Factors 1 and $3,+.03$; and Factors 2 and $3,-.08$. In contrast to the purely artifactual outcome of an orthogonal rotation, these results offer direct empirical confirmation that the three factors were indeed quite independent of one another. It is not surprising, therefore, that the factor loadings were found to be similar to those obtained by means of the Varimax solution.

\section{IMAGERY VARIABLES ANALYSIS}

Because the childhood memory variables were found to be loaded on a separate factor, Lorenz and Neisser (1985) decided to undertake an additional factor analysis of the imagery variables alone. A principal components analysis of the seven relevant measures was used to extract three factors, and Varimax rotation yielded factors that were identified as follows: Factor 1, vividness and control; Factor 2, spatial manipulation; and Factor 3, spontaneous elaboration. Table 2 shows the results of an identical analysis carried out upon the relevant correlation coefficients contained in Lorenz and Neisser's Table 2, and, once again, allowing for rounding errors, these results confirm the findings presented in their original paper. Nevertheless, the same three issues need to be addressed in evaluating these results.

With regard to the number of factors to be extracted, Lorenz and Neisser gave no justification whatsoever for the decision to identify three factors, although they mentioned that the point of the analysis was "to test for the presence of the elaboration factor isolated by Garro (1983), which had not appeared in the first analysis" (p. 497). In other words, the choice of three factors was motivated by a priori theoretical expectations rather than by independent criteria or rules of thumb. Indeed, on their own earlier account, only three factors had been suggested by "inspection of the pattern of significant correlations," including the factor relating to childhood memories, and so there could be no justification for searching for a third imagery factor. With regard to the more commonly used rules of thumb, the eigenvalues for successive factors obtained from principal components analysis of the seven variables were $2.56,1.81, .92, .61, .49, .40$, and .22 . While Cattell's (1966) scree test suggests that the choice of three factors might conceivably be appropriate, Kaiser's eigenvalue-one criterion indicates that only two factors should be extracted.

The latter conclusion was confirmed by the fact that a common factor analysis that attempted to extract three factors from these variables failed to achieve a coherent mathematical solution. Specifically, after $\mathbf{4 5}$ iterations the estimated communality of one of the variables exceeded the logical upper bound of 1.0, a so-called Heywood case. As McDonald (1985) noted, "The most common cause of Heywood cases seems to be failure on the part of the investigator to represent each factor by at least three tests with large loadings on it"' (p. 226; see also pp. 79-80). Obviously, such a situation would be an inevitable consequence of seeking to measure three (relatively independent) putative factors on the basis of only seven variables.

With regard to the initial estimates of communality, five of the seven SMCs for the imagery variables were less than .5 , which again points to the appropriateness of common factor analysis rather than principal components analysis for these data. Table 2 therefore shows the results of Varimax rotation of two factors extracted by means of common factor analysis from the relevant correlation coeffi-

Table 2

Results of Varimax Principal Components Analysis (PCA) and of Varimax and Oblimin Common Factor Analysis (CFA) of Imagery Variables

\begin{tabular}{|c|c|c|c|c|c|c|c|}
\hline & \multicolumn{3}{|c|}{ Varimax PCA } & \multicolumn{2}{|c|}{ Varimax CFA } & \multicolumn{2}{|c|}{ Oblimin CFA } \\
\hline & 1 & 2 & 3 & 1 & 2 & 1 & 2 \\
\hline Gordon (1949) & $.81 *$ & .13 & .16 & $.69 *$ & .18 & $.70^{*}$ & .24 \\
\hline Betts (1909) & $.81^{*}$ & -.25 & .17 & $.84^{*}$ & -.12 & $.83 *$ & -.05 \\
\hline Marks $(1972,1973)$ & $.78 *$ & .11 & -.01 & $.54^{*}$ & .08 & $.54^{*}$ & .13 \\
\hline Richardson (1977a) & .08 & $.85^{*}$ & .22 & .02 & $.76^{*}$ & .08 & $.76^{*}$ \\
\hline Bennett et al. (1947) & -.05 & $.85^{*}$ & .04 & -.11 & $.58 *$ & -.06 & $.57^{*}$ \\
\hline Barratt (1953) & .15 & $.52 *$ & $.74 *$ & .27 & $.82 *$ & .33 & $.84 *$ \\
\hline Richardson (1977b) & .12 & .03 & $.93 *$ & .29 & .42 & .33 & .44 \\
\hline
\end{tabular}

*Loadings greater than .50 were used to interpret the factors. 
cients. The overall impression of the factor loadings is similar to that obtained from the two relevant factors in the corresponding panel of Table 1 .

The final analysis considered whether an oblique rotation might be more appropriate for these data. Table 2 shows the structure factor matrix from an oblimin rotation carried out upon the two factors extracted by means of common factor analysis. The intercorrelation between these two factors was +.17 , confirming their relative independence, and the factor loadings were similar to those obtained in the Varimax solution.

\section{DISCUSSION}

Lorenz and Neisser (1985) summarized their results in the following manner: "Our data confirm the distinction between reported vividness of mental imagery on the one hand and objectively measured spatial visualization on the other. In addition, we confirmed the existence of a third imagery dimension, spontaneous elaboration" (p. 499). I have argued that these conclusions were obtained on the basis of inadequate analytic techniques. Nevertheless, are they upheld by the additional analyses provided in this paper?

First, all of the analyses replicated the first two factors that were identified by Lorenz and Neisser, and the oblique solutions confirmed that these two factors were relatively independent of one another. Moreover, these analyses approximately replicated the distinction noted in previous factor-analytic studies between subjective judgments in questionnaires on experienced imagery and objective performance in tests of spatial ability (Di Vesta et al., 1971; Forisha, 1975; Richardson, 1977a). An apparent exception to this among the tests used by Lorenz and Neisser was Barratt's (1983) Visualization Form, which provided ratings of the use of visualization while solving the previously completed Differential Aptitude Test and Cube Task. These responses seem to constitute introspective judgments of the vividness of experienced imagery, and yet they loaded on the spatial manipulation factor and were wholly unrelated to the vividness and control factor. One possibility is that the two factors should instead be identified, respectively, with the use of generic images stored in a long-term semantic memory and the use of specific images stored in an episodic or working memory. Another possibility (U. Neisser, personal communication, September 23, 1987) is that subjects base their responses to Barratt's Visualization Form upon their objective success or subjective dexterity in the relevant tasks, rather than upon the phenomenal qualities of their mental imagery. Further research is definitely needed to resolve the apparently anomalous status of the Barratt task.

Second, there were no adequate grounds for identifying an additional imagery factor, and indeed no factorial solution involving three factors was possible in the case of the imagery variables. In this case, Lorenz and Neisser had wished to explore the idea of individual differences in visual elaboration, which was originally put forward by Slee (1980). However, the psychometric scale devised by Slee appears never to have been properly documented; the only previous study to have linked it to a separate imagery factor was an unpublished dissertation (Garro, 1983), and its reliability and construct validity have been seriously questioned (Rooney, 1984). Lorenz and Neisser excluded it from their battery of tests, but they did include the three measures with the next highest loadings on the relevant factor in Garro's analysis: Gordon's (1949) test of imagery control, Richardson's (1977b) Verbalizer-Visualizer Questionnaire, and the Space Relations Form T of the Differential Aptitude Test (Bennett et al., 1947). Even setting aside misgivings about the use of the principal components analysis in Lorenz and Neisser's study, it must be noted that the putative third imagery factor showed loadings of only .155 and .037 on the first and third of these measures, which scarcely counts as a replication of Garro's investigation. In short, the notion of spontaneous elaboration as a distinctive imaginal trait seems wholly gratuitous.

\section{REFERENCES}

BARRATt, P. E. (1953). Imagery and thinking. Australian Journal of Psychology, 5, 154-164.

Bennett, G. K., Seashore, H. G., \& Wesman, A. G. (1947). Differential Aptitude Test: Space Relations, Form T. New York: Psychological Corporaton.

BETTS, G. H. (1909). The distribution and functions of mental imagery. New York: Teachers College.

Carroll, J. B. (1957). Biquartimin criterion for rotation to oblique simple structure in factor analysis. Science, 126, 1114-1115.

Cattell, R. B. (1966). The scree test for the number of factors. Multivariate Behavioral Research, 1, 245-276.

CATtELl, R. B. (1978). The scientific use of factor analysis in behavioral and life sciences. New York: Plenum Press.

Di Vesta, F. J., INGersoll, G., \& Sunshine, P. (1971). A factor analysis of imagery tests. Journal of Verbal Learning \& Verbal Behavior, 10, 471-479.

Forisha, B. D. (1975). Mental imagery and verbal processes: A developmental study. Developmental Psychology, 11, 259-267.

GARRO, L. C. (1983). Imagery's role in cognition: Integrating imagery theory with an individual differences approach (Doctoral dissertation, Duke University, 1982). Dissertation Abstracts Intermational, 43, 2371B.

GoRDON, R. (1949). An investigation into some of the factors that favour the formation of stereotyped images. British Journal of Psychology, 39, 156-167.

KAISER, H. F. (1960). The application of electronic computers to factor analysis. Educational \& Psychological Measurement, 20, 141-151.

LORENZ, C., \& NeISSER, U. (1985). Factors of imagery and event recall. Memory \& Cognition, 13, 494-500.

MARKS, D. F. (1972). Individual differences in the vividness of visual imagery and their effect on function. In P. W. Sheehan (Ed.), The function and nature of imagery (pp. 83-108). New York: Academic Press.

MARKS, D. F. (1973). Visual imagery differences in the recall of pictures. British Journal of Psychology, 64, 17-24.

MCDonALD, R. P. (1985). Factor analysis and related methods. Hillsdale, NJ: Erlbaum.

NoRUSIS, M. J. (1986). SPSS/PC+ advanced statistics. Chicago, IL: SPSS.

PaIvio, A. (1971). Imagery and verbal processes. New York: Holt, Rinehart \& Winston.

Richardson, A. (1969). Mental imagery. New York: Springer.

RichaRDsON, A. (1972). Voluntary control of the memory image. In P. W. Sheehan (Ed.), The function and nature of imagery (pp. 109-129). New York: Academic Press.

RichARDSON, A. (1977a). The meaning and measurement of memory imagery. British Journal of Psychology, 68, 29-43.

RichARDSON, A. (1977b). Verbalizer-visualizer: A cognitive dimension. Journal of Mental Imagery, 1, 109-126.

RoONEY, P. J. (1984). Individual differences in imagery and their relation to visual memory (Doctoral dissertation, Cornell University, 1984). Dissertation Abstracts Intermational, 45, 1608B.

Rummel, R. J. (1970). Applied factor analysis. Evanston, IL: Northwestern University Press.

SheEhan, P. W. (1967). A shortened form of Betts' Questionnaire Upon Mental Imagery. Journal of Clinical Psychology, 23, 386-389.

SLEE, J. A. (1980). Individual differences in visual imagery ability and the retrieval of visual appearances. Journal of Mental Imagery, 4, 93-113.

(Manuscript received for publication February 16, 1988.) 